\title{
Tragic Outcome in Pregnancy
}

Dr. Tahmina Begum ${ }^{1 *}$, Dr. MD. Nazrul Islam ${ }^{2}$, Dr. Ferdousi Begum ${ }^{3}$, Dr. Mahbubur Rahman ${ }^{4}$

${ }^{1}$ Associate Professor, Department of OBS \& Gynae, Holy Family Red Crescent Medical College Hospital, Dhaka, Bangladesh

${ }^{2}$ Registrar, Department of Medicine, Holy Family Red Crescent Medical College \& Hospital, Dhaka, Bangladesh

${ }^{3}$ Assistant Professor, Department of Obstetrics \& Gynaecology, Holy Family Red Crescent Medical College \& Hospital, Dhaka, Bangladesh

${ }^{4}$ Associate Professor, Department of Gynae, Patuakhali Medical College, Patuakhali, Bangladesh

DOI: $10.36347 /$ sjams.2020.v08i10.024

| Received: 30.09.2020 | Accepted: 13.10.2020 | Published: 21.10.2020

*Corresponding author: Dr. Tahmina Begum

Abstract

In this study our main goal is to the objective of this study is to understand the incidence, socio epidemiological and etiological factors of intrauterine fetal death. This reparative study was conducted in the gynae and obstetric department of Mymensingh Medical College Hospital and Holy Family Red Crescent Medical College Hospital of Dhaka City from November 2018 to October 2019. A total 1736 pregnancy were studied. Out of them 48 we intrauterine fetal death (IUFD). Early identifying of underlying risk factors \& diagnosis and timely intervention in the form of medical or surgical treatment will definitely help in reducing the morbidity and mortality of intrauterine fetal demise.

Keywords: Ectopic Pregnancy, techicardia, hypotention, cervix.

Copyright $(\mathcal{C} 2020$ The Author(s): This is an open-access article distributed under the terms of the Creative Commons Attribution 4.0 International License (CC BY-NC 4.0) which permits unrestricted use, distribution, and reproduction in any medium for non-commercial use provided the original author and source are credited.

\section{INTRODUCTION}

Fetal death is an obstetric death accounting for approximately half of perinatal death. Stillbirth is an event which has always challenged the obstetricians. The mode of antenatal \& the intrapartum surveillance for fetal wellbeing has advanced in last few decades [1]. There are so many maternal conditions and diseases that are responsible for poor obstetrical outcomes. Stillbirth is an useful index to measure the values of antenatal and intranatal care. By proper antenatal check-up and to identify the high-risk cases associated with poor outcomes can be sorted. Many patients take admission in tertiary hospitals, among them $72 \%$ are multigravida and only $22 \%$ are primigravida [2]. These cases are being reported as there are some cases having definite causes that might be prevented, as well as some other cases with unexplained causes having no symptoms ever before the tragic incident. The aim of this study was to analyze the maternal conditions associated with fetal death with specific reference to clinical presentations, fetal, and maternal complications and to find the preventable causes of fetal death [3].

IUFD a tragic event for both parents occurs in both early and late pregnancy. Perception of fetus within the uterus for several days or months when not realised by the patient which might be due to IUFD, because of deprivation of oxygen of umbilical cord or during difficult delivery process. The death is indicated by the fact that after such expulsion or extraction, the fetus doesn't breath or show any other evidence of life, such as beating of the heart, pulsation of the umbilical cord or definite movement of voluntary muscle. The heart beat is to be distinguished from the transient cardiac contraction; respirations are to be distinguished from fleeting respiratory efforts or gasps. Some other IUFD might occur even before delivery. Death of the fetus during delivery is termed as 'still born' and when fetus die in the uterus before labour begins. It can be expressed in another way like this, death of the fetus within the uterus after the age of viability or after 20 weeks of pregnancy. Patient may not realise the event or the bad thing may come in seen suddenly when the doctor examines. Sometimes patient feels fetal movement immediately before delivery. In this case, fetus can die during delivery occurs due to asphyxia or difficult labour stress. According to the Centre for Disease Control and Prevention fetal death occurs in 26 out of every thousand births of Bangladesh. One in every 200 pregnant women in developed countries was reported to have a still born baby after 28 weeks of gestation. About one in every four IUFD is due to of unknown aetiology. Causes of fetal death includes-

\section{Maternal}

Prolonged pregnancy (>42 wks.), Diabetes (poorly controlled), Systemic lupus erythematosus, Antiphospholipid syndrome, Infection, Hypertension, 
Preeclampsia, Eclampsia, Hemoglobinopathy, Advanced maternal age, $\mathrm{Rh}$ disease, Uterine rupture, Maternal trauma or death, Inherited thrombophilias [8].

\section{Fetal}

Multiple gestations, Intrauterine growth restriction, Congenital abnormality, Genetic abnormality, Infection (i.e. parvovirus B19, CMV, Listeria), Hydrops

\section{Placental}

Cord accident, Abruption, Premature rupture of membranes, Vasa previa, Fetomaternal hemorrhage, Placental insufficiency

\section{Risk factors}

Advanced maternal age, previous history of fetal demise, Maternal infertility, History of small for gestational age infant, Obesity, Paternal age.

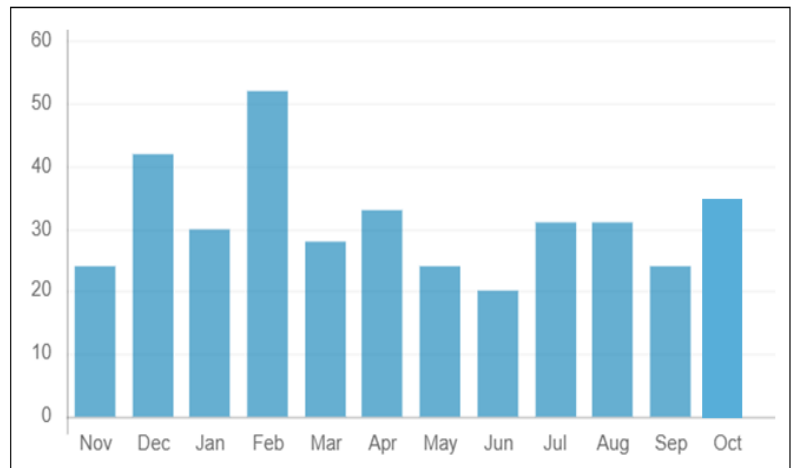

Fig-1: Intrauterine fetal death from November 2018 to October 2019

\section{CASE Reports}

A 29 years old primigravida lady was referred to our 500 bedded specialized hospital from outside private chamber with absence of fetal movement for 2 days. She had raised blood pressure. She was not a booked case and was in antenatal checkup irregularly. Regarding family history both of her parents were hypertensive.

She also was a hypothyroid since last 2 years and was on medication. She also had a history of diarrhea and vomiting for 5 days. According to LMP her EDD was on $9^{\text {th }}$ September, 2019

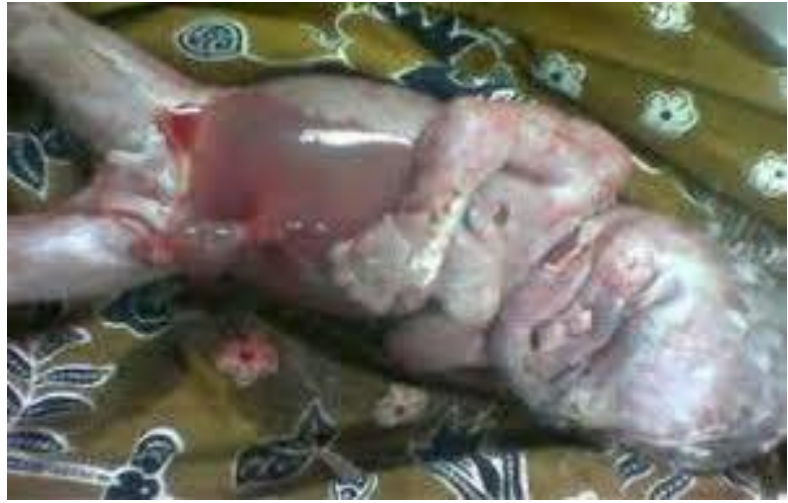

Fig-2: Macerated still born fetus

All modalities under general examination revealed no abnormalities except raised blood pressure of 200/120 mmHg, she was mildly anemic and edematous. Per abdominal examination revealed symmetrical enlargement of abdomen about $28 \mathrm{~cm}$ which was slightly smaller than her gestational age. Fetal movement was absent and no fetal heart sound was audible even with Doppler machine. Her serum uric acid level was $5.6 \mathrm{mg} / \mathrm{ml}$, alkaline phosphatase was 116.0 U/L, LDH $590 \mathrm{U} / \mathrm{L}$ and coagulation profile was within normal limit. Other blood reports revealed no abnormalities. Ultrasonogram of abdomen reported 28 weeks of pregnancy with IUFD.

Another 25 years old second gravida was hospitalized at her 39 weeks of pregnancy with absence of fetal movement for 1 day. She had lower abdominal pain for 4 hours. No fetal heart sound was audible with Doppler machine after admission. Ultrasonogram was done immediately that revealed intrauterine death, hence was diagnosed as a case of intrauterine fetal death of 37 weeks of maturity. On abdominal examination uterus was about full term in size with cephalic presentation and head was engaged, hence diagnosed as second gravida 39 weeks of pregnancy with IUFD with unexplained etiology.

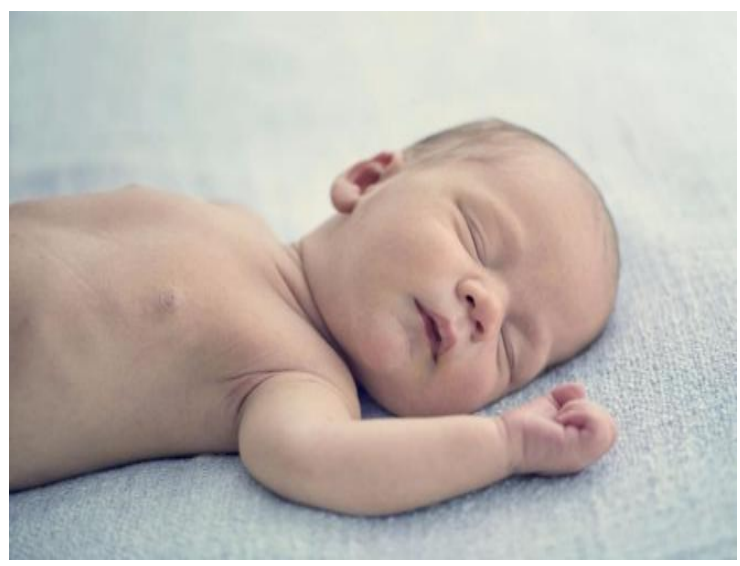

Fig-3: Fresh still born baby 


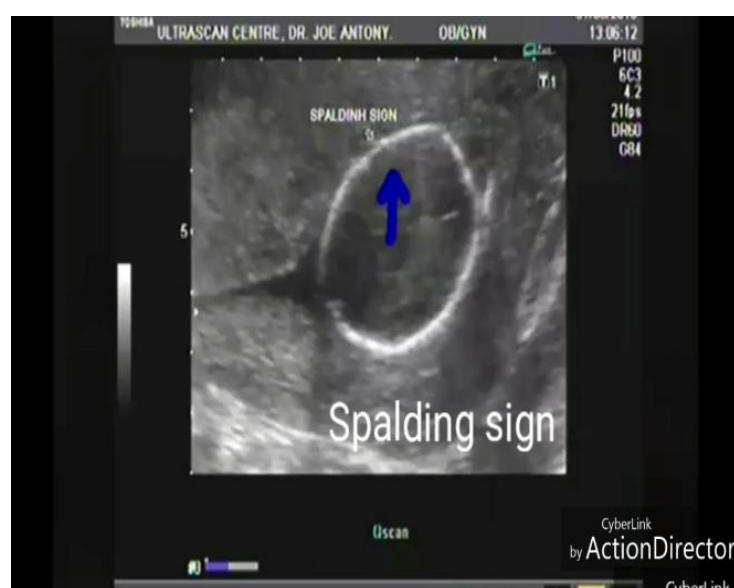

Fig-4: USG Showing spalding sign of skull bone

\section{DISCUSSION}

The etiology of fetal demise is unknown in 25$60 \%$ of all case [4]. In cases where a cause is clearly identified, the cause of fetal death can be attributable to fetal, maternal, or placental pathology. One prospective study attributed $64.9 \%$ of fetal death to placental pathology overall [5]. The same study noted higher rates of fetal demise secondary to placental pathology at late gestational age.

A meta-analysis of 96 population-based studies found that maternal overweight and obesity was the highest-ranking modifiable risk factor for stillbirth [6]. Advanced maternal age ( $>35 \mathrm{y}$ ) and maternal smoking were also significant [7]. Small size for gestational age and abruption were the highest-ranking pregnancy disorder risk factors for stillbirth. Obstetric conditions and placental abnormalities were the most common causes of stillbirth in one population-based study [8]. Preexisting diabetes and hypertension are also important contributors to stillbirth.

Once the diagnosis of fetal demise has been confirmed it is extremely difficult for both the family and the health care providers. In this stressful time it is important to ensure that the emotional needs of the family are met. The patient should be informed of her condition [9]. Often, allowing the mother to see the lack of cardiac activity helps her to accept the diagnosis.

Termination of pregnancy should be offered after diagnosis some wish to begin induction immediately, while others wish to delay induction for a period of hours or days until they are emotionally prepared [10].

A useful resource is a grief packet that can be given to the parents following the demise. This usually includes referrals for counseling, support groups, and other resources [11]. A container or folder can be included so that the family can preserve keepsakes such as photos, footprints, or a lock of hair. Spiritual support is an important resource during such difficult times and should always be offered to patients and their families [12].

\section{CONCLUSiON}

Hypertensive disorder in pregnancy or family history of hypertension or any crisis in pregnancy such as acute vomiting of diarrhea, high fever should be carefully evaluated. The possibility of vascular disease or other hematological causes or electrolyte imbalance should be excluded. These patients should be dealt with multidisciplinary team to optimize this tragic outcome and ensure comprehensive management. Since strong family history of hypertension is less responsive to treatment, early identification and management of crisis is recommended. Improved application of current knowledge may help to decrease the fetal death rate caused by fetal growth retardation. Awareness of hypertension, diabetes mellitus, thyroid dysfunctions, history of congenital abnormalities and other risk factors can play a major role to prevent such mishap therefore contribute to build a healthier future generation.

\section{REFERENCE}

1. World Health Organization. More Women Worldwide Receive Early Antenatal Care, But Great Inequalities Remain. 2017. Retrieved from https://www.who.int/reproductivehealth/early-ancworldwide/en/

2. No GTG. Late intrauterine fetal death and stillbirth. 2010.

3. Mattingley P. Evaluation of Fetal Death: Definition of Fetal Death, Frequency of Fetal Death, Diagnosis of Fetal Death. Medscape [Internet], 2016; 1-12.

4. Korteweg FJ, Erwich JJ, Holm JP, Ravisé JM, van der Meer J, Veeger NJ, Timmer A. Diverse placental pathologies as the main causes of fetal death. Obstetrics \& Gynecology. 2009 Oct 1;114(4):809-17.

5. Flenady V, Koopmans L, Middleton P, Frøen JF, Smith GC, Gibbons K, Coory M, Gordon A, Ellwood D, McIntyre HD, Fretts R. Major risk factors for stillbirth in high-income countries: a systematic review and meta-analysis. The lancet. 2011 Apr 16;377(9774):1331-40.

6. Marufu TC, Ahankari A, Coleman T, Lewis S. Maternal smoking and the risk of still birth: systematic review and meta-analysis. BMC public health, 2015; 15(1):239.

7. Danielsson K. Very Well Family. Causes and Risk of Stillbirth. 2019. Retrieved From https://www.verywellfamily.com/intrauterinefetal-demise-2371631

8. Preboth M. American Academy of Family Physicians. ACOG Guidelines on Antepartum Fetal Surveillance. 2000. Retrieved From https://www.aafp.org/afp/2000/0901/p1184.html

9. Manning FA, Levine D. Biophysical profile test for antepartum fetal assessment. 
10. Gardosi J, Madurasinghe V, Williams M, Malik A, Francis A. Maternal and fetal risk factors for stillbirth: population based study. Bmj. 2013 Jan $24 ; 346$.

11. Reddy UM, Goldenberg R, Silver R, Smith GC, Pauli RM, Wapner RJ, Gardosi J, Pinar H, Grafe M, Kupferminc M, Varli IH. Stillbirth classification-developing an international consensus for research: executive summary of a
National Institute of Child Health and Human Development workshop. Obstetrics and gynecology. 2009 Oct;114(4):901.

12. Reddy UM, Page GP, Saade GR, Silver RM, Thorsten VR, Parker CB, Pinar H, Willinger M, Stoll BJ, Heim-Hall J, Varner MW. Karyotype versus microarray testing for genetic abnormalities after stillbirth. New England Journal of Medicine. 2012 Dec 6;367(23):2185-93. 